\title{
The impact of poloidal asymmetries on tungsten transport in the core of JET H-mode plasmas
}

\author{
C. Angioni ${ }^{1}$, F. J. Casson ${ }^{2}$, P. Mantica ${ }^{3}$, T. Pütterich ${ }^{1}$, M. Valisa ${ }^{4}$, E. \\ A. Belli ${ }^{5}$, R. Bilato ${ }^{1}$, C. Giroud ${ }^{2}$, P. Helander ${ }^{6}$, and JET contributors * \\ EUROfusion Consortium, JET, Culham Science Centre, Abingdon, OX14 3DB, UK \\ ${ }^{1}$ Max-Planck-Institut für Plasmaphysik, D-85748 Garching, Germany \\ ${ }^{2}$ CCFE, Culham Science Centre, Abingdon, OX14 3DB, UK \\ ${ }^{3}$ Istituto di Fisica del Plasma, CNR/ENEA, Milano, Italy \\ ${ }^{4}$ Consorzio RFX-CNR/ENEA, I-35127 Padova, Italy \\ 5 General Atomics, PO Box 85608, San Diego, CA 92186-5608, USA \\ ${ }^{6}$ Max-Planck Institut für Plasmaphysik, Greifswald, Germany
}

\begin{abstract}
Recent progress in the understanding and prediction of the tungsten behaviour in the core of JET H-mode plasmas with ITER-like wall is presented. Particular emphasis is given to the impact of poloidal asymmetries of the impurity density. In particular it is shown that the predicted reduction of temperature screening induced by the presence of low field side localization of the tungsten density produced by the centrifugal force is consistent with the observed tungsten behaviour in a JET discharge in H-mode baseline scenario. This provides first evidence of the role of poloidal asymmetries in reducing the strength of temperature screening. The main differences between plasma parameters in JET baseline and hybrid scenario discharges which affect the impact of poloidally asymmetric density on the tungsten radial transport are identified. This allows the conditions by which tungsten accumulation can be avoided to be more precisely defined.
\end{abstract}

PACS numbers: 52.25.Fi, 52.55.Fa, 52.65.Tt

\footnotetext{
* See the Appendix of F. Romanelli et al., Proceedings of the 25th IAEA Fusion Energy Conference 2014, San Petersburg, Russia
} 


\section{INTRODUCTION}

Since 2011, JET moved to an ITER-like wall (ILW), which features W (atomic number 74 , atomic mass 183.84) as the plasma facing material in the divertor, and Be in the main chamber walls $[1,2]$. This, in combination with the recent decision to start ITER operation directly with a $\mathrm{W}$ divertor, has strongly motivated studies dedicated to the investigation, the modelling and the prediction of the $\mathrm{W}$ behaviour in JET-ILW plasmas in different operational regimes and discharge scenarios [3-6]. Clear evidence has been produced that in the core of JET-ILW plasmas, W transport is dominated by neoclassical transport $[4,5]$. Thereby, limitation or avoidance of $\mathrm{W}$ accumulation can be obtained by different methods which aim at reducing the dominance of the neoclassical inward convection, that is the pinch, driven by the main ion density gradient.

This can be obtained in several ways.

- A reduction of the central peaking of the main ion density profile, possibly in combination with the increase of the peaking of the main ion temperature profile, which enhances the favorable effect of the temperature screening.

- A reduction of the overall magnitude of the neoclassical transport, in particular by a reduction of the usual low field side (LFS) localization of the $\mathrm{W}$ density produced by centrifugal $(\mathrm{CF})$ effects $[7,8]$, which is known to produce an enhancement of the neoclassical transport [9-13]. This can be obtained by a reduction of the plasma toroidal rotation, or by the impact of auxiliary heating systems. In particular, ion cyclotron resonance heating $(\mathrm{ICRH})$ produces a temperature anistropy of the resonant minority species, which consequently develops a poloidally asymmetric density distribution and impacts the poloidal asymmetry of the background electrostatic potential, as well as the asymmetry of the $\mathrm{W}$ density [14-17]. With LFS ICRH resonance, this can reduce the LFS localization of W, or even induce a moderate HFS-LFS asymmetry (provided plasma rotation is small enough), accompanied by a significant reduction of the neoclassical transport [12]. Moreover, strong temperature gradients of the minority which are produced by localized ICRH power absorption can also have a favorable effect by providing an additional contribution to the temperature screening, at least in regions where their effective temperature is not too high [5].

- An increase of the turbulent transport, for instance by application of additional central auxiliary heating, which can reduce the dominant impact of the neoclassical convection. In contrast to neoclassical transport, turbulent impurity transport does not exhibit convective mechanisms which increase with increasing charge or mass of the heavy impurity [18-20]. However, it is not strongly modified by poloidal asymmetries $[21,22]$, therefore in the presence of strong rotation, it can become relatively small as compared to the neoclassical transport component over a large radial window at the plasma center [4].

In a previous work [4] we have focused on the $\mathrm{W}$ behaviour in hybrid scenarios in the presence of neutral beam injection (NBI) heating only. This first investigation was motivated by the regular strong accumulation observed in this scenario. We have shown that the time evolution of the $\mathrm{W}$ density in the core of these plasmas closely follows the time evolution of the background density profiles, as it is determined by the neoclassical convection in the region inside $r / a \simeq 0.3$. That work also provided a fairly complete description of the $\mathrm{W}$ transport in hybrid scenarios and demonstrated the quantitative agreement with the 

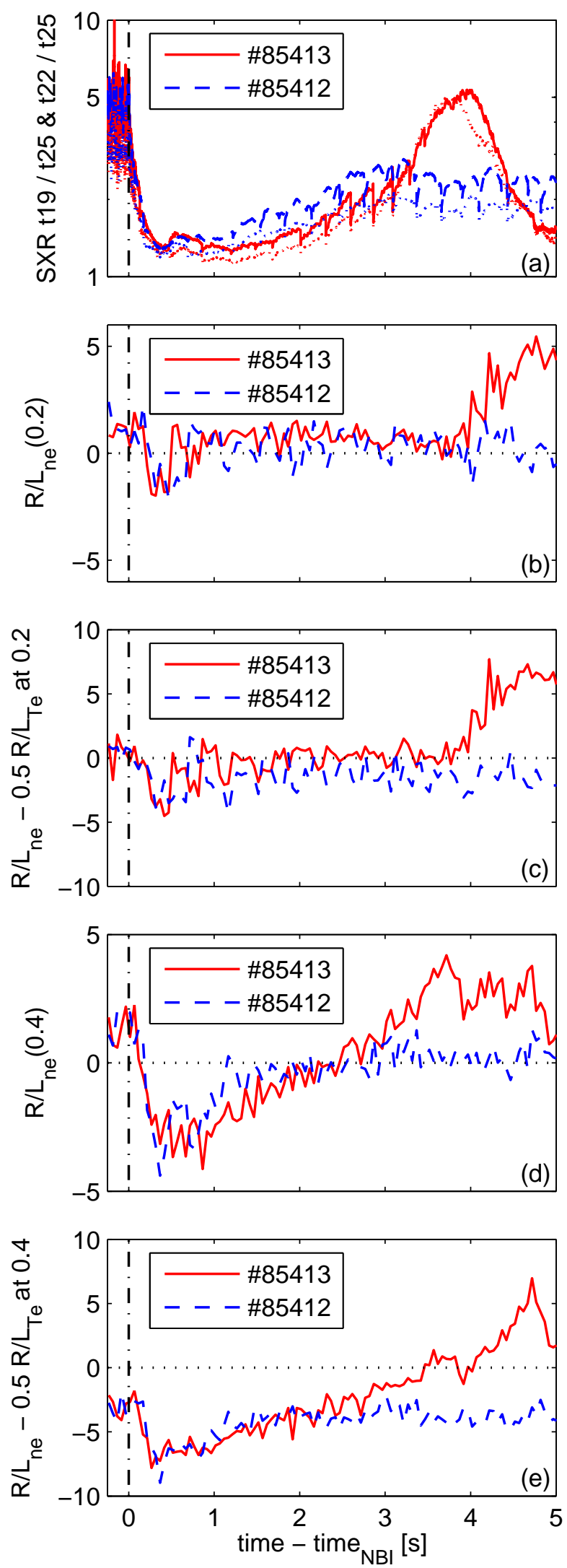

FIG. 1: Time evolution of the central LOS of the vertical soft X-ray (SXR) camera $\left(t_{19}\right)$, divided by the LOS tangent to the surface $\mathrm{r} / \mathrm{a}=0.4\left(t_{25}\right)$, as well as of the LOS tangent to the surface $\mathrm{r} / \mathrm{a}=0.22\left(t_{22}\right)$ divided by the LOS $t_{25}$ (dotted lines) (a). Time evolution of the normalized logarithmic density gradient $R / L_{n}$ at $\mathrm{r} / \mathrm{a}=0.2$ (b) and $\mathrm{r} / \mathrm{a}=0.4(\mathrm{~d})$, as well as of the PfirschSchlüter convection parameter $R / L_{n}-0.5 R / L_{T}$ at $\mathrm{r} / \mathrm{a}=0.2$ (c) and $\mathrm{r} / \mathrm{a}=0.4$ (e) from a pair of JET discharges in baseline scenario at 2.6 MA and $2.7 \mathrm{~T}$ [6], $\sharp 85413$ (solid, 20MW NBI) and $\sharp 85412$ (dashed, 16MW NBI $+4 \mathrm{MW} \mathrm{ICRH).} \mathrm{Measurements} \mathrm{of} \mathrm{the} \mathrm{electron} \mathrm{density} \mathrm{and} \mathrm{temperature} \mathrm{have}$ been used as proxy for the deuterium density and temperature. 
experiment obtained with a combination of neoclassical and turbulent $\mathrm{W}$ transport models which include the impact of poloidal asymmetries on the $\mathrm{W}$ transport. However, it also showed that, in the experimental conditions of hybrid scenarios, the inclusion of poloidal asymmetries does not deliver results which are qualitatively different from those obtained by a model which neglects their impact. Thereby, that previous study was not able to provide experimental evidence of the modification of $\mathrm{W}$ transport produced by poloidal asymmetries.

Another previous study has focused on the impact of localized ICRH [5] in the H-mode baseline scenario. There it has been shown that, in addition to the indirect impact that ICRH can have on the $\mathrm{W}$ behaviour connected with the modification of the background plasma profiles (in particular the decrease of the central density peaking and the decrease of the plasma rotation), the are direct effects connected with the development of a population of energetic ions of the ICRH minority which have different perpendicular and parallel temperatures, leading to a modification of the poloidal asymmetry of $\mathrm{W}$ which, in particular, is predicted to affect the magnitude of the neoclassical transport [12]. It has been also shown that localized heating can also develop strong gradients of the minority temperature which have an impact on the $\mathrm{W}$ behaviour by means of an additional contribution to the temperature screening [5].

In this work, we focus on the impact of poloidal asymmetries produced by toroidal rotation in H-modes in baseline scenario, with NBI heating only. Baseline scenarios are regularly operated at higher current with respect to hybrid scenarios, with values of edge safety factor $q_{95} \simeq 3$ in contrast to hybrid scenarios with edge safety factor $q_{95} \simeq 4$. In addition, hybrid scenarios have regularly lower densities and higher values of normalized plasma pressure $\beta_{N}=\langle\beta\rangle /\left(I_{p} / a B\right)$ (where $\langle\beta\rangle$ is the volume average of $2 \mu_{0} p / B^{2}$ measured in $\%$, $a$ is the minor radius in $\mathrm{m}, \mathrm{B}$ is the magnetic field in $\mathrm{T}$ and $I_{p}$ is the plasma current in MA) [24]. The interest of the experimental conditions regularly achieved in baseline scenarios is that the $\mathrm{W}$ accumulation is usually determined in a radial window which is not as centrally localized as observed in hybrid scenarios, where the behaviour of the $\mathrm{W}$ transport is tracked by the behaviour of the background density and temperature profiles very close to the magnetic axis $(r / a \leq 0.3)$.

This is shown in Fig. 1, where the time evolution of an approximate proxy for the $\mathrm{W}$ peaking is compared to the time evolution of the density and temperature gradients of the main plasma. We consider the time evolution of a pair of discharges at 2.6 MA and $2.7 \mathrm{~T}$, one which exhibits W accumulation ( $\sharp 85413$ with $20 \mathrm{MW}$ of $\mathrm{NBI}$ ) and one which does not exhibit accumulation ( $\sharp 85412$ with $16 \mathrm{MW}$ of NBI and $4 \mathrm{MW}$ of ICRH) [6]. In Fig. 1(a) we observe that the ratio between the central line of sight (LOS) of the soft X-ray $(\mathrm{SXR})$ vertical camera [23] $\left(t_{19}\right)$ and a more peripheral LOS $\left(t_{25}\right)$ (tangent to the magnetic surface $r / a=0.4$ ) follows the same time evolution of the ratio of the LOS $t_{22}$ (tangent to the magnetic surface $r / a=0.2$ ) and $t_{25}$. This suggests that the accumulation is not occuring with the same extreme central localization which was observed in hybrid scenarios [4]. Consistent with these considerations on the radial window relevant for the $\mathrm{W}$ accumulation, we observe that the time evolutions of these proxies for the $\mathrm{W}$ peaking, that is the ratios $t_{19} / t_{25}$ and $t_{22} / t_{25}$ follow the time evolution of the normalized logarithmic density gradient $R / L_{n}=-(R / n)(d n / d r)$ as well as that of the neoclassical impurity convection parameter $R / L_{n}-0.5 R / L_{T}$ at $r / a=0.4$. A clear difference between the two discharges with and without accumulation is observed in the time traces of the gradients of density and temperature at this radial location, which tracks the different behaviour of the SXR signals. This is consistent with the results of modelling of this pair of pulses presented in [6] which show the largest differences in the theoretically predicted convection to diffusion 

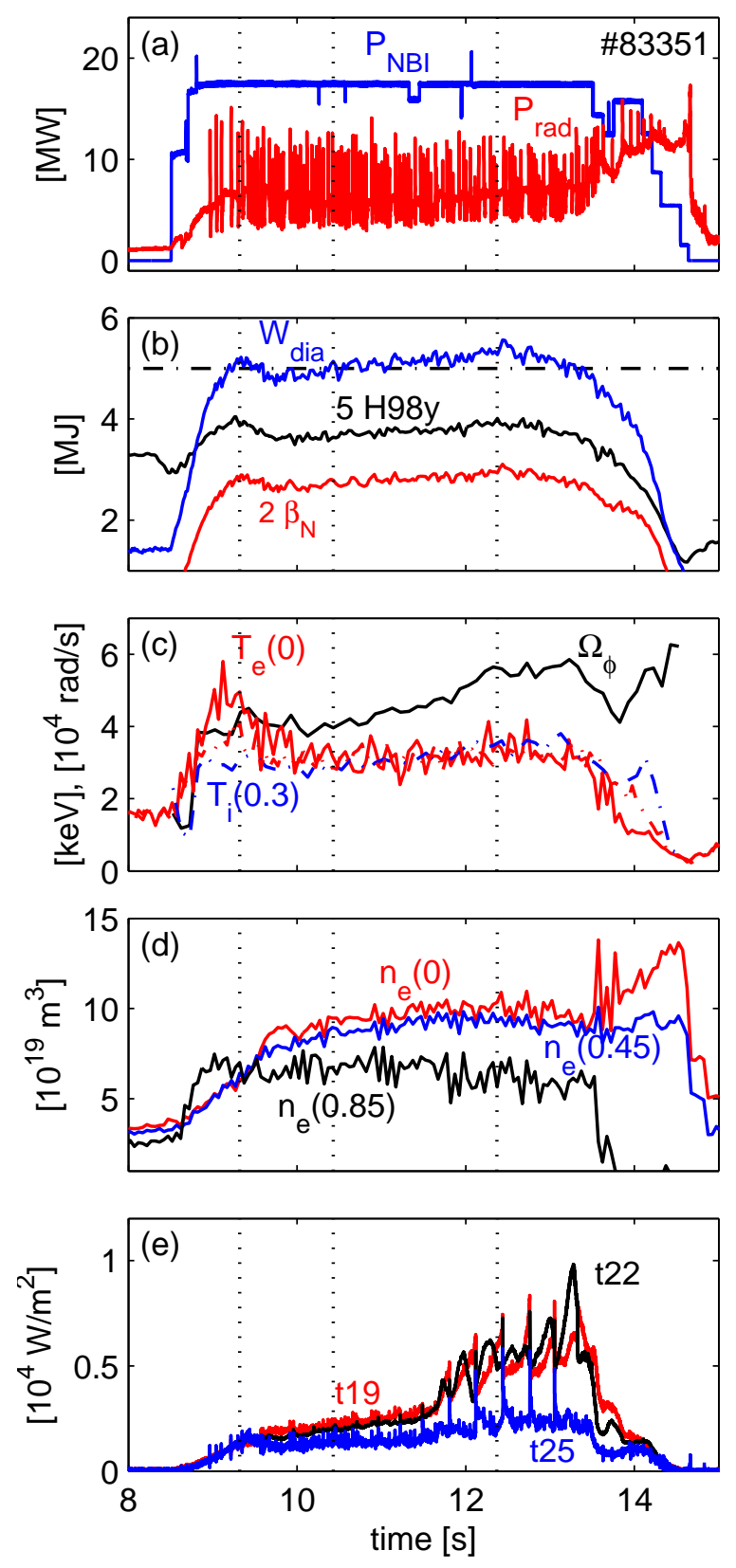

FIG. 2: Time traces of (a) the NBI and the radiated powers, (b) the total stored energy $W_{\text {dia }}$ in MJ, the normalized pressure parameter $\beta_{N}$ (times 2), and the H98y factor (times 5), (c) the electron temperature at $r / a=0$ and $r / a=0.3$ (dash-dotted), as well as the ion (carbon) temperature (at $r / a=0.3)$ and central toroidal rotation, (d) the central, intermediate $(r / a=0.45)$ and peripheral (pedestal top $r / a=0.85$ ) density, (e) the central ('t19'), intermediate ('t22', chord tangent to magnetic surface $\mathrm{r} / \mathrm{a}=0.22$ ) and peripheral (chord tangent to magnetic surface $\mathrm{r} / \mathrm{a}=0.4$ ) lines of sight of the SXR emission, during the high power phase of JET-ILW baseline scenario shot \#83351. Vertical dotted lines mark the time slices at which specific analysis and theory-based modelling have been performed. 
ratio between $0.3 \leq r / a \leq 0.5$ (Fig. 28 of that Reference). In contrast, no clear difference is observed in the behaviour of the two discharges at $r / a=0.2$ up to 4 s after the switch on of the NBI power (after $4 \mathrm{~s}$, the total radiated power of $\sharp 85413$ starts to increase, central electron temperature drops as do the central SXR signals, and also the density profile starts to peak in the center, due to the decrease of net central heating power, which reinforces the accumulation process [6]). We conclude that, in contrast to hybrid scenarios, where the accumulation takes place inside $r / a=0.3$ and is governed by the density peaking in that very central region (Fig. 1(e), Fig. 19 and Fig. 20 in [4]), in baseline scenario the W accumulation is usually governed by a wider and less central radial window $0.3<r / a<0.5$.

Since the poloidal asymmetry of the impurity density scales as $\epsilon M^{2}$ (where $\epsilon$ is the inverse aspect ratio and $M$ is the thermal Mach number), we expect that effects on transport produced by poloidal asymmetries become stronger when the $\mathrm{W}$ behaviour is determined by the behaviour of the background density profiles in this more external region. In addition, baseline scenario plasmas achieve higher collisionalities and are in the collisionality window in which changes of sign of the impurity convection produced by poloidal asymmetries, as predicted in the Pfirsch-Schlüter regime $[11,12]$ can take place, whereas these are less significant at the lower collisionalities of hybrid scenarios (as is demonstrated in Section 3, and already pointed out in [5]).

All these elements motivate the present study dedicated to the time evolution of the $\mathrm{W}$ behaviour in a baseline JET-ILW H-mode, in order to identify specific theoretically predicted effects of the $\mathrm{W}$ poloidal asymmetry on the $\mathrm{W}$ radial transport, and validate these effects against experimental measurements. To this end, we have modelled three time slices and compared the predictions with and without the inclusion of CF effects. We shall show that the neglect of $\mathrm{CF}$ effects leads to an overestimate of the impact of temperature screening, and does not allow us to reproduce the experimental W behaviour, which exhibits W accumulation.

The organization and approach of the present study closely follows that of [4], and this reference will be regularly considered in order to develop a comparison of the $\mathrm{W}$ behaviour in the two scenarios, as already carried out in this section. The remainder of this paper is organized as follows. In the next section we present the plasma discharge which has been considered for the analysis, and we describe the experimental evidence for the process of $\mathrm{W}$ accumulation. In section 3, the physical picture of the impact of $\mathrm{W}$ density asymmetry on the $\mathrm{W}$ transport is briefly reviewed by means of an analytical model [11, 12], and the numerical results obtained with the drift kinetic neoclassical code NEO [25, 26] will be interpreted through the understanding provided by the analytical model. In Section 4, the modelling results, which also include the contribution of turbulent $W$ transport, computed with the gyrokinetic code GKW [27, 28], are compared with the experimentally reconstructed W density distribution, as obtained with an interpretive 2D W density diagnostic based on the SXR signals $[4,29]$. Section 5 presents the main conclusions of this work, and places them into the context of recent other studies dedicated to $\mathrm{W}$ transport in JET-ILW.

\section{EXPERIMENTAL OBSERVATIONS}

The time traces of the JET-ILW discharge $\sharp 83351$ are presented in Fig. $2 .\left(B_{T}=2.5 \mathrm{~T}\right.$, $I_{p}=2.75 \mathrm{MA}$, low triangularity). With respect to the fast accumulation process displayed by the hybrid scenarios, in baseline scenarios the accumulation can be mitigated or even avoided thanks to the more regular presence of sawtooth activity $[6,30]$. However, although the total radiated power remains constant over a large fraction of the high power phase of the discharge 
(a), the signal of the central lines of sight (LOS) of the vertical SXR camera (both $t_{19}$, close to the magnetic axis, and $t_{22}$, tangent to the magnetic flux surface $r / a=0.22$ on the low field side (LFS)) show a progressive increase (modulated by the sawtooth crashes) with respect to a more peripheral LOS ( $t_{25}$ tangent to the magnetic flux surface $r / a=0.4$ on the LFS) in (e)). In agreement with the analysis presented in the previous section, and in contrast to a similar analysis performed on the time evolution of a discharge in hybrid scenario $(\$ 82722$ [4]) we observe that the radial window where the accumulation takes place is usually wider in the baseline scenario than in the hybrid scenario. We select three time slices representative of the time evolution of this discharge. These will be considered for the calculation of $\mathrm{W}$ transport, combining the results of the NEO and GKW codes. The first, at $9.32 \mathrm{~s}$, is during the early times of the high power phase, when the electron density profile is still hollow. The second, at $10.42 \mathrm{~s}$, is at the start of the phase exhibiting central peaking of the $\mathrm{W}$ density and flat electron density profile. The third, at 12.37s, is during the $\mathrm{W}$ accumulation phase, with a centrally peaked electron density profile. The electron temperature and electron density profiles (measured by the High Resolution Thomson Scattering diagnostic) as well as the profiles of the toroidal rotation and the thermal Mach number of deuterium at these three time slices are presented in Fig. 3. The ion temperature is assumed to be equal to the electron temperature, which is consistent (within large uncertainties) with the available measurements of the ion temperature and is realistic at these high densities. From the cubic spline fits of the data, we compute the logarithmic gradients of the electron density and electron temperature, normalized to the major radius. These are presented in Fig. 4. In addition, we also show the analytical expression of the neoclassical convection to diffusion ratio when the impurity is in the Pfirsch-Schlüter regime, the background plasma is in the banana regime, and a poloidally symmetric density is assumed [31]

$$
\frac{R V_{Z}}{D_{Z}}=-Z\left(\frac{R}{L_{n}}-0.5 \frac{R}{L_{T}}\right),
$$

where density and temperature gradients refer to the main ions species, but where we have used the precise measurements of the electron density and temperature in place of less accurate measurements of the ions in Figure 3(c). We observe that with this simple analytical model (which also assumes poloidal symmetry of the $\mathrm{W}$ density) the $\mathrm{W}$ convection is directed outward over a large portion of the minor radius for all of the three time slices, at least in the interval $0.2<r / a<0.8$ and even in radial windows where the values of $R / L_{n}$ are positive, which implies a dominant role of the temperature screening. This can be expected to prevent $\mathrm{W}$ accumulation in all of the three time slices, in contrast with the observation shown in Fig. 1. However, this simple analysis neglects the impact of the presence of a poloidal asymmetry of the $\mathrm{W}$ density, which will be considered in the next section by means of calculations with the NEO code.

\section{REDUCTION OF TEMPERATURE SCREENING INDUCED BY THE POLOIDAL ASYMMETRY OF THE W DENSITY}

Theoretical works have pointed out that the neoclassical transport of heavy impurities can be strongly modified in the presence of poloidal asymmetries of the impurity density [9-13]. Here we recall the result of [11] which has been recently extended in [12], considering the impact of arbitrary poloidal asymmetry, and not only that arising from CF effects. We 
express the results of those previous works in the following general form,

$$
\frac{R\left\langle\Gamma_{Z}^{n e o}\right\rangle}{\left\langle n_{Z}\right\rangle}=q^{2} D_{c} Z\left[\left(\frac{1}{Z} \frac{R}{L_{n Z}}-\frac{R}{L_{n i}}+\frac{1}{2} \frac{R}{L_{T i}}\right) P_{A}-0.33 P_{B} f_{c} \frac{R}{L_{T i}}\right]
$$

where, consistently with the definitions introduced in [5], the two geometrical coefficients are

$$
\begin{gathered}
P_{A}=\frac{1}{2 \epsilon^{2}} \frac{\left\langle B^{2}\right\rangle}{\left\langle n_{Z}\right\rangle}\left[\left\langle\frac{n_{Z}}{B^{2}}\right\rangle-\left\langle\frac{B^{2}}{n_{Z}}\right\rangle^{-1}\right], \\
P_{B}=\frac{1}{2 \epsilon^{2}} \frac{\left\langle B^{2}\right\rangle}{\left\langle n_{Z}\right\rangle}\left[\frac{\left\langle n_{Z}\right\rangle}{\left\langle B^{2}\right\rangle}-\left\langle\frac{B^{2}}{n_{Z}}\right\rangle^{-1}\right],
\end{gathered}
$$

and where $f_{c}$ is the fraction of circulating particles and \langle\rangle denotes the flux surface average. In Eq. (2) $D_{C}=\rho_{i}^{2} / \tau_{i i}$ is the classical diffusion coefficient, with $\rho_{i}^{2}=v_{t h i}^{2} /\left\langle\Omega_{i}^{2}\right\rangle$ the fluxsurface averaged ion Larmor radius squared, and $\tau_{i i}=3(2 \pi)^{3 / 2} \epsilon_{0}^{2} \sqrt{m_{i}} T_{i}^{3 / 2} /\left(n_{i} e^{4} \ln \Lambda\right)$ the ion-ion collision time.

The geometrical factor $P_{A}$ generalizes the usual Pfirsch-Schlüter factor in the case of poloidally asymmetric heavy impurity density. In the poloidally symmetric case and in the large aspect ratio limit, $P_{A}=1$. The coefficient $P_{B}$ is equal to zero in the poloidally symmetric case, but it is non-zero in the poloidally asymmetric case, and arizes from the presence of a poloidal rotation in combination with a poloidally inhomogeneous impurity density. It provides a component which is also proportional to the logarithmic ion temperature gradient, and therefore modifies the relative strength of the temperature screening effect. In the limit of infinitely strong poloidal localization of the impurity density, $P_{A}=P_{B}=0.5 \epsilon^{-2}$ [12]. This implies that the strong localization of impurities has two unfavorable consequences, the strong increase of the neoclassical transport and the reduction of the relative weight of the temperature screening.

In order to quantitatively take into account these theoretically expected effects, we apply the drift-kinetic code NEO $[13,25]$ to the three time slices, and we compare the results obtained with and without the inclusion of the poloidal asymmetry of the $\mathrm{W}$ density produced by $\mathrm{CF}$ effects. In these calculations, a single $\mathrm{W}$ species is considered, with a charge which changes along the minor radius and is given by the local average over the concentrations of all the $\mathrm{W}$ ionization stages. This radially varying averaged charge is around 46 in the center, and decreases to 34 at the edge. The results are presented in Fig. 5, where open and full symbols show the results of the calculations without and with the inclusion of $\mathrm{CF}$ effects, respectively. The numerical results reproduce the expectations derived from the analytical model Eq. (2). In Fig. 5(a) we observe the strong increase of the neoclassical impurity diffusivity in the presence of poloidal asymmetries with respect to the results obtained assuming poloidal symmetry. Both diffusion and convection are increased by a similar amount, therefore the convection to diffusion ratio exhibits more limited variations. The poloidally symmetric results predict a convection directed outward for $r / a>0.2$ for all the three time slices, qualitatively consistent with the simple analytical model for poloidal symmetry of Eq. (1) and with the curves of Fig. 3(c). In contrast, the results which include the impact of poloidal asymmetries produced by CF effects exhibit a clear reduction of the convection with respect to the poloidally symmetric case. In the case of the intermediate and late time slices, this produces a change of sign, yielding a pinch in some radial windows, and in particular a pinch in the radial window $r / a \geq 0.3$ at $\mathrm{t}=12.37 \mathrm{~s}$. 
The prediction of a reversal of the neoclassical convection from outward to inward as a consequence of the inclusion of CF effects is a result of extreme interest, because it provides a qualitatively different prediction with and without the inclusion of poloidal asymmetries. This different predicted behaviour was not obtained in the work dedicated to the modelling of hybrid scenarios [4], mainly because of the combination of two effects. In hybrid scenarios the collisionality is lower, and impurities are not as deep in the Pfirsch-Schlüter regime as in the baseline scenario. In addition, in hybrid scenario $\mathrm{W}$ accumulation is mainly determined by the behaviour of the background density and temperature profiles in the most central region $(r / a<0.3)$ where little or no difference is obtained in the predictions of the convection to diffusion ratios with and without the inclusion of $\mathrm{CF}$ effects. In fact, in hybrid scenario the inclusion of $\mathrm{CF}$ effects impacts the prediction of the convection to diffusion ratio only close to the edge (Fig. 4 of [4]). In contrast, in baseline scenario W accumulation is regularly governed by the behaviour of the background profiles in a less central region $(0.3<$ $r / a<0.5)$, where the impact of the inclusion of CF effects is significantly stronger at the collisionalities achieved in baseline scenario.

The demonstration that in the numerical results this difference has to be entirely ascribed to the reduction of the temperature screening coefficient, as predicted by the analytical model (2), is provided in Fig. 6. When the temperature screening effect is removed, by removing the ion temperature gradient in the NEO calculations, the predicted convection to diffusion ratios with and without $\mathrm{CF}$ effects are practically identical.

This qualitative difference in the prediction of the sign of the neoclassical convection with and without the inclusion of the impact of poloidal asymmetries (and in the presence of a non-zero ion temperature gradient) allows us to experimentally validate this important piece of theory, which affects our capability of quantitatively predicting the impact of the temperature screening. As already mentioned in Section I, this validation was not possible in [4], since at the plasma parameters of the hybrid scenario no significant difference is found between the ratio of neoclassical convection to diffusion with and without the inclusion of the impact of poloidal asymmetries. The main cause for this difference in the plasma parameters of the two scenarios is given by the higher collisionality in baseline scenario, which brings W in the Pfirsch-Schlüter regime over a larger portion of the minor radius. The critical role of collisionality is demonstrated in Fig. 7, where the NEO calculations have been performed using as inputs the same background gradients as those of Fig. 5 (from measurements in $\sharp 83351$ ), but where the collisionality has been taken from the hybrid discharge $\sharp 82722$ modelled in [4] (which has a ion-ion collision frequency which is about 3 times smaller). We observe that at the lower collisionality of the hybrid scenario, only limited differences are found between the results with (full symbols) and without (open symbols) the inclusion of $\mathrm{CF}$ effects. In fact, with decreasing collisionality, the plasma region where the difference in $R V_{W N E O} / D_{W N E O}$ with and without the inclusion of $\mathrm{CF}$ effects becomes significant is more and more peripheral. This identifies collisionality as the critical parameter in order to obtain an experimentally measurable difference in the predictions.

In order to proceed to a comparison with the experimentally reconstructed two dimensional (2D) W density distribution over the poloidal cross section, the turbulent transport has to be added to the neoclassical transport. The turbulent transport contributions have been computed with the GKW code $[27,28]$ by means of linear gyrokinetic calculations over a spectrum from $k_{y} \rho_{i}=0.15$ to $k_{y} \rho_{i}=0.7$, where $k_{y}$ is the binormal wave number and $\rho_{i}=\sqrt{T_{i} / m_{i}} / \Omega_{c i}$. As in the NEO calculations, a single $\mathrm{W}$ species is considered, with a radially varying charge. The sum of neoclassical and turbulent transport components is performed following the modelling approach described in [4] (in particular in Eq. 3), 
which assumes stationary and source free conditions. Therefore, in order to compute the logarithmic density gradient of the LFS W density profile, we use the following equation,

$$
\frac{R}{L_{n W}}=-\frac{\frac{\chi_{i a n}}{\chi_{i \mathrm{NEO}}} \frac{R V_{W \mathrm{GKW}}}{\chi_{i \mathrm{GKW}}}+\frac{R V_{W \mathrm{NEO}}}{\chi_{i \mathrm{NEO}}}}{\frac{\chi_{i a n}}{\chi_{i \mathrm{NEO}}} \frac{D_{W \mathrm{GKW}}}{\chi_{i \mathrm{GKW}}}+\frac{D_{W \mathrm{NEO}}}{\chi_{i \mathrm{NEO}}}} .
$$

In Eq. (3) the turbulent ion heat conductivity predicted by the GK calculations $\chi_{i \mathrm{GKW}}$ is assumed to match the anomalous part of the ion heat conductivity $\chi_{i a n}=\chi_{i P B}-\chi_{i N E O}$. Here $\chi_{i P B}$ is the power balance ion heat conductivity obtained by interpretive simulations with the JETTO transport code [32], whereas $\chi_{i N E O}$ is the neoclassical ion heat conductivity. The terms at the numerator and the denominator of Eq. (3) obtained with NEO and GKW are shown in Figs. 8, 9, 10, where full and open symbols present the results obtained with and without the inclusion of poloidal asymmetry effects respectively. Similar to the results obtained in [4] and [5], we find that the effects of the poloidal asymmetry are relatively more limited on the turbulent transport components, as theoretically expected [21, 22], and an additional consequence of the inclusion of the poloidal asymmetry is the increase of the relative weight of the neoclassical component of transport (which is strongly enhanced by the poloidal asymmetry) with respect to the turbulent transport component.

The profiles of the LFS logarithmic density gradients are computed with Eq. (3), and are shown in Fig. 11(a), again, with (full symbols) and without (open symbols) the inclusion of CF effects. The correspoding integrated LFS density profiles are shown in Fig. 11(b), normalized to the value at the boundary of the radial integration domain $(r / a=0.85)$. The different directions of the neoclassical convection obtained with and without the inclusion of $\mathrm{CF}$ effects in the intermediate and late time slices are reflected in the different signs of the LFS $R / L_{n W}$ and the qualitatively different profile shapes of the LFS W density, clearly presented in Fig. 11(b). These differences are outside the uncertainties of the experimentally reconstructed $\mathrm{W}$ density. Therefore, we expect a comparison with the experimental measurements to provide clear evidence of the experimental relevance of the impact of poloidal density asymmetries on transport.

\section{COMPARISON WITH 2D SXR W DENSITY DIAGNOSTIC}

The experimental 2D W density distribution over the poloidal cross section has been reconstructed adopting an interpretive diagnostic based on the SXR signals [29]. A description of this diagnostic technique can be found in [4]. Fig. 12 shows the comparison of the predicted and the experimental $\mathrm{W}$ density profiles as a function of the major radius along a cut at the height of the magnetic axis. The integration constants of the predicted density profiles have been set in order to match the maximum experimental $\mathrm{W}$ density. For comparison, also the profiles of the $\mathrm{W}$ densities predicted without the inclusion of $\mathrm{CF}$ effects are presented (dashed lines). Here the same value of the density at the boundary as that of the profiles obtained with the inclusion of CF effects has been used, for direct comparison between the two predictions. The predicted profiles including the poloidal asymmetry effects are found to be in quantitative agreement with the experiment, within the experimental uncertainties. In contrast, while for the first time slice, the result obtained without $\mathrm{CF}$ effects is still in qualititative agreement with the experiment, though poloidally symmetric, for the intermediate and the late time slices the simulations performed neglecting the $\mathrm{CF}$ effects are in clear (qualitative) disagreement with the experimental results. This result provides clear evidence (for the first time to our knowledge in a comparison between 
theoretical predictions and measurements) that poloidal asymmetries can play a critical role in affecting transport, and their neglect can lead to even qualitatively wrong predictions, that is to predict absence of accumulation, while $\mathrm{W}$ accumulation is taking place in reality, as in Fig. 12(c) (3rd time slice). The comparison with the experimentally reconstructed W density can be also performed directly considering the entire 2D poloidal distribution of the $\mathrm{W}$ density. The contour plots of the 2D W density distributions are shown in Fig. 13.

\section{CONCLUSIONS}

The study presented in this paper adds a new and important element in the ongoing activity of understanding and predicting the $\mathrm{W}$ behaviour in JET plasmas $[4,5]$.

In [4], we have established the theoretical modelling approach and demonstrated the dominance of neoclassical transport, strongly enhanced by CF effects, in driving the $\mathrm{W}$ accumulation, particularly in JET H-modes in hybrid scenario. The modelling was shown to closely reproduce the experimentally reconstructed $\mathrm{W}$ density, providing an unprecendented validation of a modelling approach which consistently includes the impact of poloidal asymmetries in both the neoclassical and turbulent transport components for the prediction of the $2 \mathrm{D} \mathrm{W}$ density distribution over the poloidal cross section [4].

In [5], we have extended the modelling approach in order to also include the impact of the presence of an energetic minority species heated by ICRH and therefore characterized by a temperature anisotropy [5]. There we have demonstrated that, in the case of centrally localized ICRH power deposition, the presence of the minority impacts $\mathrm{W}$ transport providing not only an additional source of poloidal asymmetry, but also providing an additional component to the temperature screening, caused by collisions between $\mathrm{W}$ and the minority ions in the presence of very strong temperature gradients of the effective temperature of the minority.

In the present work, we have focused on the impact of poloidal asymmetries produced by toroidal rotation in JET-ILW H-modes in baseline scenario, with NBI heating only. This study has allowed us to validate an additional critical piece of the theory of neoclassical transport in the presence of poloidal asymmetries, specifically connected with the reduction of the effect of temperature screening in the presence of poloidal localization of the W density [11-13]. We have shown that, similarly to the hybrid scenario, the $\mathrm{W}$ accumulation process in the baseline scenario is mainly governed by neoclassical transport, and follows the time evolution of the background density profile. However, in contrast to the hybrid scenario, the radial region which has strongest impact on the $\mathrm{W}$ accumulation process is around $0.3 \leq r / a \leq 0.5$, and therefore more external with respect to the hybrid scenario, where the $\mathrm{W}$ accumulation is governed by the behaviour of the background density inside $r / a=0.3$ [4]. These different regions likely reflect the different causes connected with the development of the density peaking in the relevant radial windows. In high density H-modes in baseline scenario, the Ware pinch appears to play the dominant role [6]. In H-modes in hybrid scenarios, at lower density and also with deeper penetration of the NBI fuelling, the localized central peaking of the plasma density, occurring very close to the magnetic axis, is likely caused by the impact of the particle source produced by the NBI in combination with a low particle diffusion [33]. In general, low (turbulent) diffusivity of the main sepcies in the central region of the plasma allows these effects to increase the peaking of the density profile, with unfavorable consequences on the $\mathrm{W}$ behaviour. This is confirmed by the observation that a reduction of net central heating, as it occurs due to an increase of the central radiated power, regularly leads to an increase of the central peaking of the electron density (as shown 
for instance in Fig. 1). In baseline scenario, the higher collisionality, in combination with the increased importance of the neoclassical transport in a more peripheral region with respect to the hybrid scenario, allows the poloidal asymmetry of the $\mathrm{W}$ density to significantly impact the $\mathrm{W}$ transport, an aspect which was not observed in the analysis of the hybrid scenario. In contrast to the study presented in [4], where a qualititative agreement with the experimental $\mathrm{W}$ density was also demonstrated by modelling which neglects the impact of $\mathrm{CF}$ effects, in this study, dedicated to H-modes in baseline scenario, we have provided clear demonstration that the neglect of the impact of poloidal asymmetries on $\mathrm{W}$ transport can lead to a qualitatively wrong prediction, that is, a prediction of absence of accumulation when accumulation is taking place in the experiment. In agreement with past $[10,11]$ and more recent $[12,13]$ theoretical studies, the inclusion of $\mathrm{CF}$ effects and therefore of the impact of poloidal asymmetries not only produces a strong enhancement of the neoclassical transport, but also reduces the relative weight of the temperature screening convective component with respect to the density gradient driven pinch. Thereby, as demonstrated in simulations which are found to closely reproduce the experimentally observed behaviour of the $\mathrm{W}$ density, $\mathrm{W}$ accumulation can occur also in conditions when neoclassical transport theory which assumes poloidal symmetry would predict absence of $\mathrm{W}$ accumulation. The agreement between the theoretical predictions and the experimental observations also provides the first experimental validation that poloidal density asymmetries can have a significant impact on the radial transport of the impurities.

While, as demonstrated in this work, at the usually higher collisionality of baseline scenarios the temperature screening effect is more significantly reduced by $\mathrm{CF}$ effects than in lower collisionality hybrid scenario, it is also important to remind that other effects imply that the $\mathrm{W}$ behaviour in hybrid sceanrio is regularly less stable that in baseline. In particular, the presence of regular sawteeth in baseline scenario $[6,30]$ and the overall reduced values of central peaking of the background density imply that in general the $\mathrm{W}$ behaviour in baseline scenario H-modes is more stable than in hybrid scenarios. In addition, under certain conditions, the appearance of neoclassical tearing modes (NTMs), more regular at the higher $\beta$ of the hybrid scenario H-modes, can accelerate or even start the accumulation process, which still remains mainly governed by neoclassical transport [4].

In recent JET experimental campaigns $\mathrm{W}$ accumulation can be limited or avoided by the application of central ICRH $[3,5,6,36,37]$, also consistent with past experiences in C-Mod [34] and JET [35]. The main role of central ICRH is to limit the peaking of the background density profile with favorable consequences for the reduction of the neoclassical pinch. Moreover, ICRH can increase the ion temperature and reduce the plasma rotation, with beneficial effects for the $\mathrm{W}$ behaviour through an increase of the temperature screening and a reduction of the enhancement of the neoclassical transport produced by $\mathrm{CF}$ effects. Central ICRH can also increase the turbulent transport, which can balance the more dangerous neoclassical transport. Finally, it can provide direct beneficial effects through the development of ICRH minority ion species with temperature anisotropy [14, 15, 17], which can produce both an additional temperature screening [5] and reduce the neoclassical transport by reducing the rotational LFS asymmetry or even producing a moderate HFS asymmetry $[12,13]$.

In the presence of strong localized ICRH, the predicted W transport strongly depends on the detailed features of the minority temperature anisotropy profiles [5] as predicted by wave solvers coupled to Fokker Planck codes. The quantitative agreement found between theoretical predictions of $\mathrm{W}$ density and experimental measurements in the absence of ICRH as documented in the present study, as well as in the previous study [4], suggests that W density measurements in the presence of strong localized ICRH can be used as an indirect 
validation of the simulations of wave-Fokker Planck packages, since predictions from neoclassical transport theory with the inclusion of poloidal asymmetry effects have been found to closely reproduce the experimentally reconstructed $\mathrm{W}$ densities when ICRH is absent, as shown in $[4,5]$, as well as in this work. In the process to further validate and extend present modelling of heavy ion transport, an important missing element is provided by the comparison of the absolute magnitude of both diffusion and convection separately. Transient transport experiments with heavy impurity laser ablation provide the required experimental data for this type of comparisons with the theoretical predictions. First studies, where the modelling approach of [4] (and applied here) has been also applied to the modelling of laser ablation experiments, have provided a promising level of agreement [38]. Further research will be dedicated to this activity in the near future from both the experimental and the modelling sides.

\section{Acknowledgments}

This work has been carried out within the framework of the EUROfusion Consortium and has received funding from the Euratom research and training programme 2014-2018 under grant agreement No 633053. The views and opinions expressed herein do not necessarily reflect those of the European Commission.

[1] G. F. Matthews, M. Beurskens, S. Brezinsek, M. Groth, E. Joffrin, A..Loving, M. Kear, M.-L. Mayoral, R. Neu, P. Prior, V. Riccardo, F. Rimini, M. Rubel, G. Sips, E. Villedieu, P. de Vries9, M. L. Watkins1,6 and EFDA-JET contributors, Phys. Scr. 2011, 014001 (2011).

[2] R. Neu, G. Arnoux, M. Beurskens, V. Bobkov, S. Brezinsek, J. Bucalossi, G. Calabro, C. Challis, J. W. Coenen, E. de la Luna, P. C. de Vries, R. Dux, L. Frassinetti, C. Giroud, M. Groth, J. Hobirk, E. Joffrin, P. Lang, M. Lehnen, E. Lerche, T. Loarer, P. Lomas, G. Maddison, C. Maggi, G. Matthews, S. Marsen, M.-L. Mayoral, A. Meigs, Ph. Mertens, I Nunes, V. Philipps, T. Pütterich, F. Rimini,M. Sertoli, B. Sieglin, A. C. C. Sips, D. van Eester, G. van Rooij, and JET-EFDA Contributors, Phys. Plasmas 20, 056111 (2013).

[3] T. Pütterich T., R. Dux, R. Neu, M. Bernert, M. N. A. Beurskens, V. Bobkov, S. Brezinsek, C. Challis, J. W. Coenen, I. Coffey, A. Czarnecka, C. Giroud, P. Jacquet, E Joffrin, A. Kallenbach, M. Lehnen, E. Lerche, E. de la Luna, S. Marsen, G. Matthews, M.-L. Mayoral, R. M. McDermott, A. Meigs, J. Mlynar, M. Sertoli, G. van Rooij, the ASDEX Upgrade Team and JET EFDA Contributors, Plasma Phys. Control. Fusion 55, 124036 (2013).

[4] C. Angioni, P. Mantica, T. Pütterich, M. Valisa, M. Baruzzo, E.A. Belli, P. Belo, F.J. Casson, C. Challis, P. Drewelow, C. Giroud, N. Hawkes, T.C. Hender, J. Hobirk, T. Koskela, L. Lauro Taroni, C.F. Maggi, J. Mlynar, T. Odstrcil, M.L. Reinke, M. Romanelli and JET EFDA Contributors, Nucl. Fusion 54, 083028 (2014).

[5] F. J. Casson, C. Angioni, E. A. Belli, R. Bilato, P. Mantica, T. Odstrcil, T. Pütterich, M. Valisa, L. Garzotti, C. Giroud, J. Hobirk, C.F. Maggi, J. Mlynar and M.L. Reinke, Plasma Phys. Control. Fusion 57, 014031 (2015).

[6] C. Giroud, S. Jachmich, P. Jacquet, A. Jarvinen, E. Lerche, F. Rimini, L. Aho-Mantila, N. Aiba, I. Balboa, P. Belo, C. Angioni, M. Beurskens, S. Brezinsek, F.J. Casson, I. Coffey, G. Cunningham, E. Delabie, S. Devaux, P. Drewelow, L. Frassinetti, A. Figueiredo, A. Huber, 
J. Hillesheim, L. Garzotti, M. Goniche, M. Groth, H.T. Kim, M. Leyland, P. Lomas, G. Maddison, S. Marsen, G. Matthews, A. Meigs, S. Menmuir, T. Pütterich, G. van Rooij, S. Saarelma, M. Stamp, H. Urano, A. Webster and JET EFDA Contributors, Plasma Phys. Control. Fusion in press (2015) (41st EPS Conf. Plasma Phys. 2014, I1.102).

[7] F. L. Hinton and S. K. Wong, Phys. Fluids 28, 3082 (1985).

[8] J. A. Wesson, Nucl. Fusion 37, 577 (1997).

[9] S. K. Wong, Phys. Fluids 30, 818 (1987).

[10] M. Romanelli, M. Ottaviani, Plasma Phys. Control. Fusion 40, 1757 (1998).

[11] T. Fülöp T. and P. Helander, Phys. Plasmas 6, 3066 (1999).

[12] C Angioni and P. Helander, Plasma Phys. Control. Fusion 56, 124001 (2014).

[13] E. A. Belli, J. Candy and C. Angioni, Plasma Phys. Control. Fusion 56, 124002 (2014).

[14] L. C. Ingesson, H. Chen, P. Helander, and M. J. Mantsinen, Plasma Phys. Control. Fusion 42, $161(2000)$.

[15] M. L. Reinke, I. H. Hutchinson, J. E. Rice, N. T. Howard, A. Bader, S. Wukitch, Y. Lin, D. C. Pace, A. Hubbard, J. W. Hughes and Y. Podpaly, Plasma Phys. Control. Fusion 54, 045004 (2012).

[16] M. L. Reinke, I. H. Hutchinson, J. E. Rice, M. Greenwald, N. T. Howard, A. Hubbard, J. W. Hughes, J. L. Terry, S. M. Wolfe, Phys. Plasmas 20, 056109 (2013).

[17] R. Bilato, O. Maj and C. Angioni, Nucl. Fusion 54, 072003 (2014).

[18] C. Angioni C. and A. G. Peeters, Phys. Rev. Lett. 96095003 (2006).

[19] N. Dubuit, X. Garbet, T. Parisot, R. Guirlet, and C. Bourdelle, Phys. Plasmas 14, 042301 (2007).

[20] Y. Camenen, A. G. Peeters, C. Angioni, F. J. Casson, W. A. Hornsby, A. P. Snodin and D. Strintzi, Phys. Plasmas 16, 012503 (2009).

[21] C. Angioni, F. J. Casson, C. Veth and A. G. Peeters, Phys. Plasmas 19, 122311 (2012).

[22] A. Mollén, I. Pusztai, T. Fülöp Ye.O. Kazakov, Phys. Plasmas 19, 052307 (2012).

[23] B. Alper et al, Rev. Sci Instrum. 68, 778 (1997).

[24] M.N.A. Beurskens, L. Frassinetti, C. Challis, T. Osborne, P.B. Snyder, B. Alper, C. Angioni, C. Bourdelle, P. Buratti, F. Crisanti, E. Giovannozzi, C. Giroud, R. Groebner, J. Hobirk, I. Jenkins, E. Joffrin, M.J. Leyland, P. Lomas, P. Mantica, D. McDonald, I. Nunes, F. Rimini, S. Saarelma, I. Voitsekhovitch, P. de Vries, D. Zarzoso and JET-EFDA Contributors, Nucl. Fusion 53, 013001 (2013).

[25] E. Belli and J. Candy, Plasma Phys. Control. Fusion 50, 095010 (2008).

[26] E. Belli and J, Candy, Plasma Phys. Control. Fusion 54015015 (2012).

[27] A. G. Peeters, Y. Camenen, F. J. Casson, W.A. Hornsby, A. P. Snodin, D. Strintzi and G. Szepesi, Comput. Phys. C. 180, 2650 (2009).

[28] F. J. Casson, A. G. Peeters, C. Angioni, Y. Camenen, W. A. Hornsby, A. P. Snodin, and G. Szepesi, Phys. Plasmas 17, 102305 (2010).

[29] T. Pütterich T. et al, Tungsten Screening and Impurity Control in JET, IAEA Fusion Energy Conf., San Diego, EX/P3-15 (2012).

[30] P. Mantica, C. Angioni, F. J. Casson et al, Proc. 41 ${ }^{\text {th }}$ European Physical Society Conference on Plasma Physics, Berlin 2014, P1.107, http://ocs.ciemat.es/EPS2014PAP/pdf/P1.017.pdf (2014).

[31] S. P. Hirshman and D. J. Sigmar, Nucl. Fusion 21, 1079 (1981).

[32] G. Cenacchi, A. Taroni, Rapporto ENEA RT/TIB (88)5 (1988).

[33] P. Mantica et al, Proc. $40^{\text {th }}$ European Physical Society Conference on Plasma Physics, Helsinki 2013, P4.141, http://ocs.ciemat.es/EPS2013ABS/pdf/P4.141.pdf (2013). 
[34] J. E. Rice, P. T. Bonoli, E. S. Marmar, S. J. Wukitch, R. L. Boivin, C. L. Fiore, R. S. Granetz, M. J. Greenwald, A. E. Hubbard, J. W. Hughes, I. H. Hutchinson, J. H. Irby, Y. Lin, D. Mossessian, M. Porkolab, G. Schilling, J. A. Snipes and S. W. Wolfe, Nucl. Fusion 42, 510 (2002).

[35] M. F. F. Nave, J. Rapp, T. Bolzonella, R. Dux, M. J. Mantsinen, R. Budny, P. Dumortier, M. von Hellermann, S. Jachmich, H. R. Koslowski, G. Maddison, A. Messiaen, P. Monier-Garbet, J. Ongena, M. E. Puiatti, J. Strachan, G. Telesca, B. Unterberg, M. Valisa, P. de Vries and contributors to the JET-EFDA Workprogramme, Nucl. Fusion 43, 1204 (2003).

[36] M. Goniche, E. Lerche, P. Jacquet, et al, Proc. $41^{\text {th }}$ European Physical Society Conference on Plasma Physics, Berlin 2014, O4.129, http://ocs.ciemat.es/EPS2014PAP/pdf/O4.129.pdf (2014).

[37] E. Lerche et al, Proc. 25 ${ }^{\text {th }}$ IAEA Fusion Energy Conference 2014, EX/P5-39, San Petersburg, Russia.

[38] M. Valisa et al Proc. 25 $5^{\text {th }}$ IAEA Fusion Energy Conference 2014, EX/6-1, San Petersburg, Russia. 

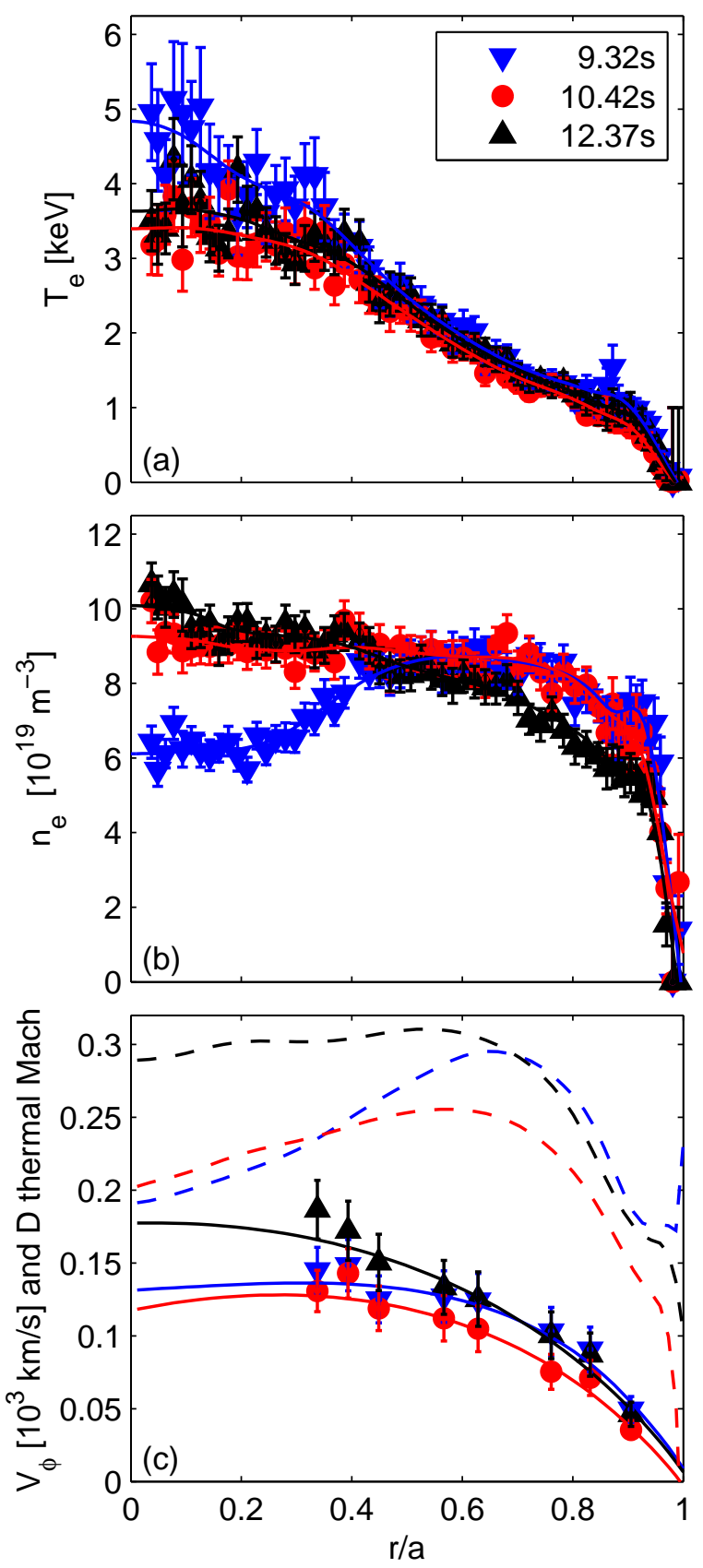

FIG. 3: The high resolution Thomson scattering raw data of electron temperature and density at three time slices quoted in the legend of shot $\sharp 83351$, plotted in symbols with error bars in (a) and (b) respectively, together with the corresponding cubic spline fits used in the modelling, and the measured toroidal rotation velocity (symbols) and corresponding fits (solid lines) with which thermal Deuterium Mach number profiles of the toroidal rotation are evaluated (dashed lines) (c). 

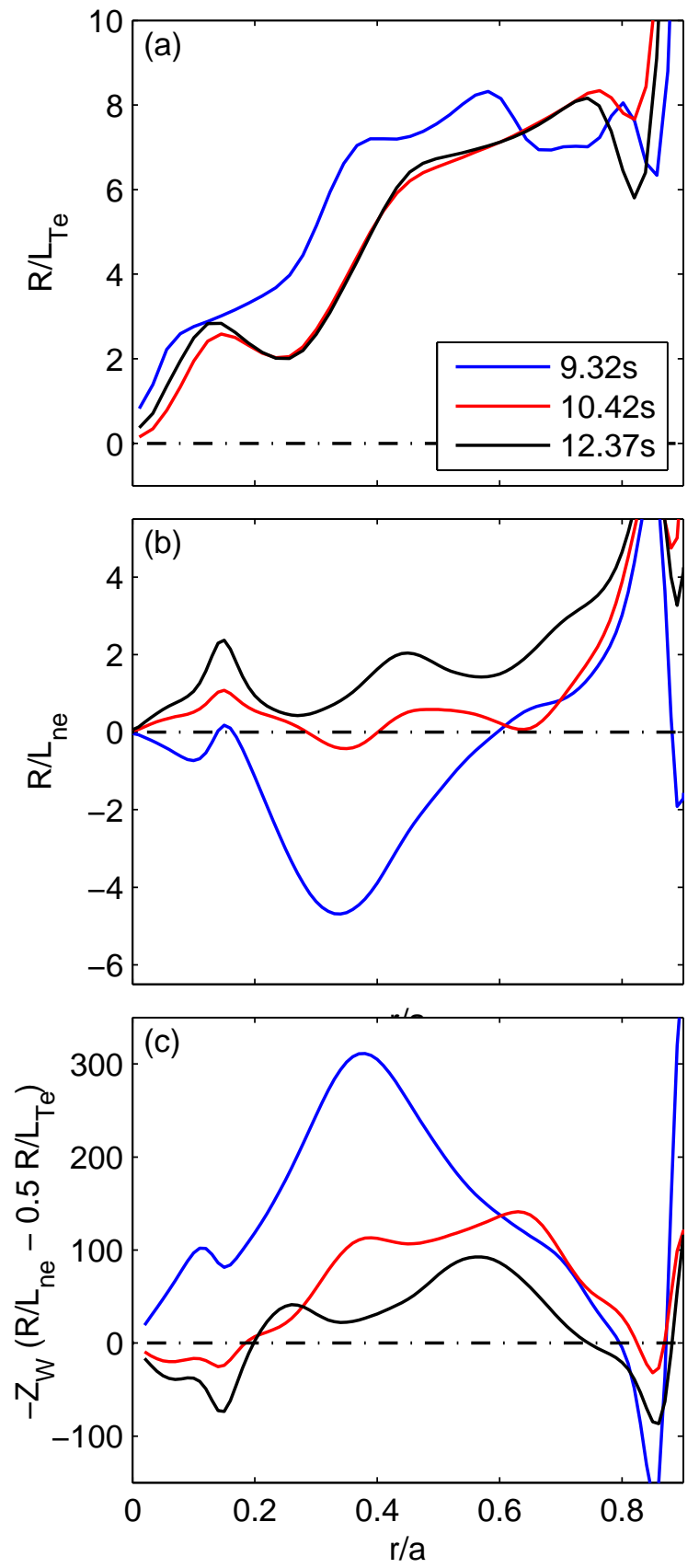

FIG. 4: Logarithmic gradients of the electron density (a) and temperature (b) normalized to the major radius $R$, as well as the Pfirsch-Schlüter convection to diffusion ratio (c), evaluated with electron density and temperature profiles as proxies for the ion density and temperature profiles. In (c) the $\mathrm{W}$ charge $Z_{W}$ is given by a radially varying charge computed as average over the charge concentrations of all the $\mathrm{W}$ ionization stages. 

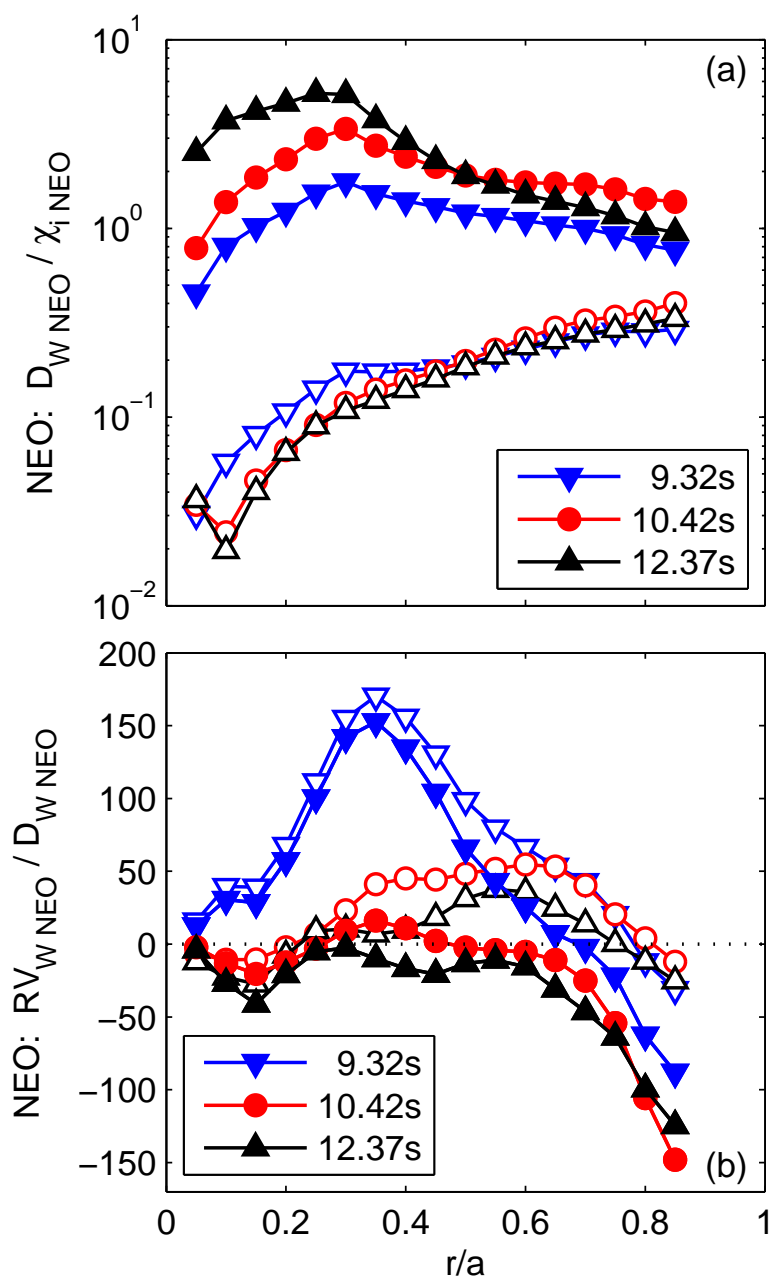

FIG. 5: Profiles of the neoclassical $\mathrm{W}$ diffusion to ion heat conductivity ratio $D_{W}$ NEO/ $\chi_{i \text { NEO }}$ (a) and the neoclassical $\mathrm{W}$ convection to diffusion ratio $R V_{W \text { NEO}} / D_{W}$ NEO (b) computed by NEO, with (full symbols) and without (open symbols) the inclusion of rotational (CF) effects, at the three time slices $9.32 \mathrm{~s}$ (triangles pointing down) and $10.42 \mathrm{~s}$ (circles) and $12.37 \mathrm{~s}$ (triangles pointing up) of $\sharp 83351$. 


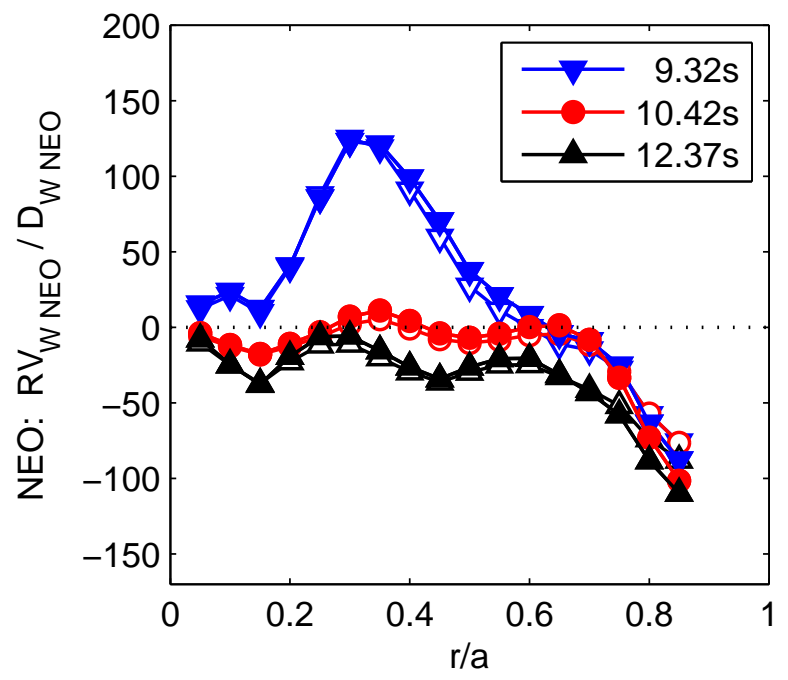

FIG. 6: Same as Fig. 5(b), this time with temperature screening effect switched off in both NEO simulations with (full) and without (open) the inclusion of CF effects 

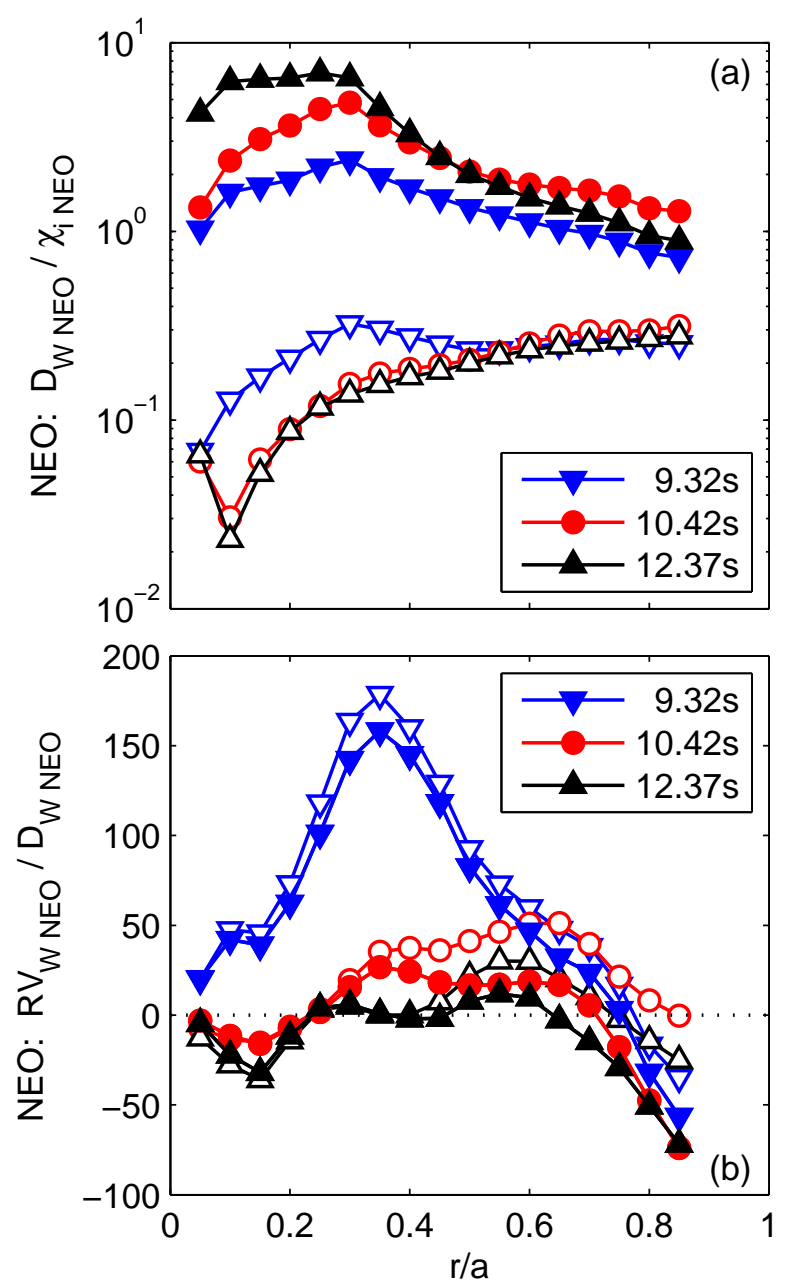

FIG. 7: Same as Fig 5, that is, profiles of the neoclassical W diffusion to ion heat conductivity ratio $D_{W \mathrm{NEO}} / \chi_{i \mathrm{NEO}}$ (a) and the neoclassical normalized $\mathrm{W}$ convection to diffusion ratio $R V_{W \mathrm{NEO}} / D_{W \mathrm{NEO}}$ (b) computed by NEO, with (full symbols) and without (open symbols) the inclusion of rotational (CF) effects, at the three time slices $9.32 \mathrm{~s}$ (triangles pointing down) and $10.42 \mathrm{~s}$ (circles) and $12.37 \mathrm{~s}$ (triangles pointing up) of $\sharp 83351$, but with reduced collisionality, which has been set as input to be equal to that of the hybrid scenario discharge $\sharp 82722$. 

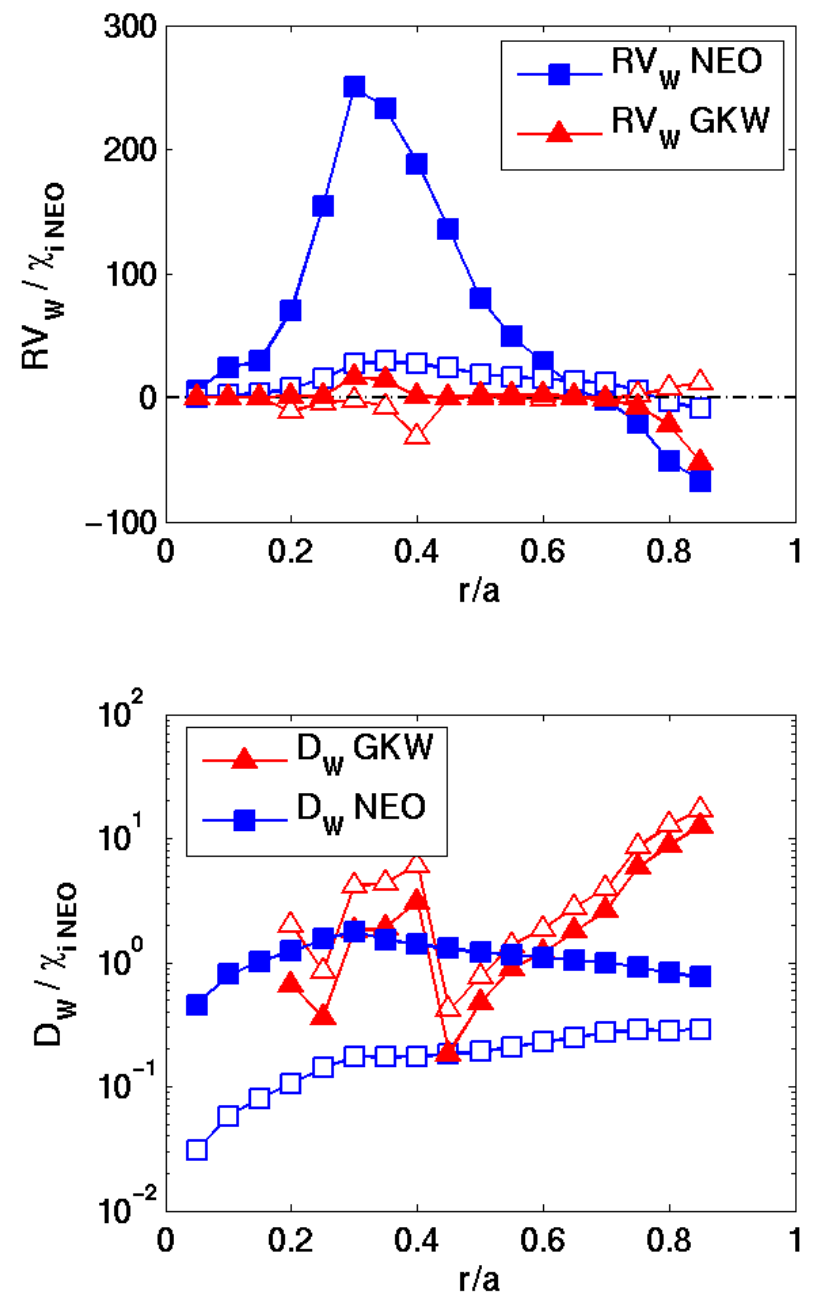

FIG. 8: W neoclassical and turbulent transport contributions computed by NEO and GKW respectively as they appear in Eq. (3) for the time slice at 9.32s. 

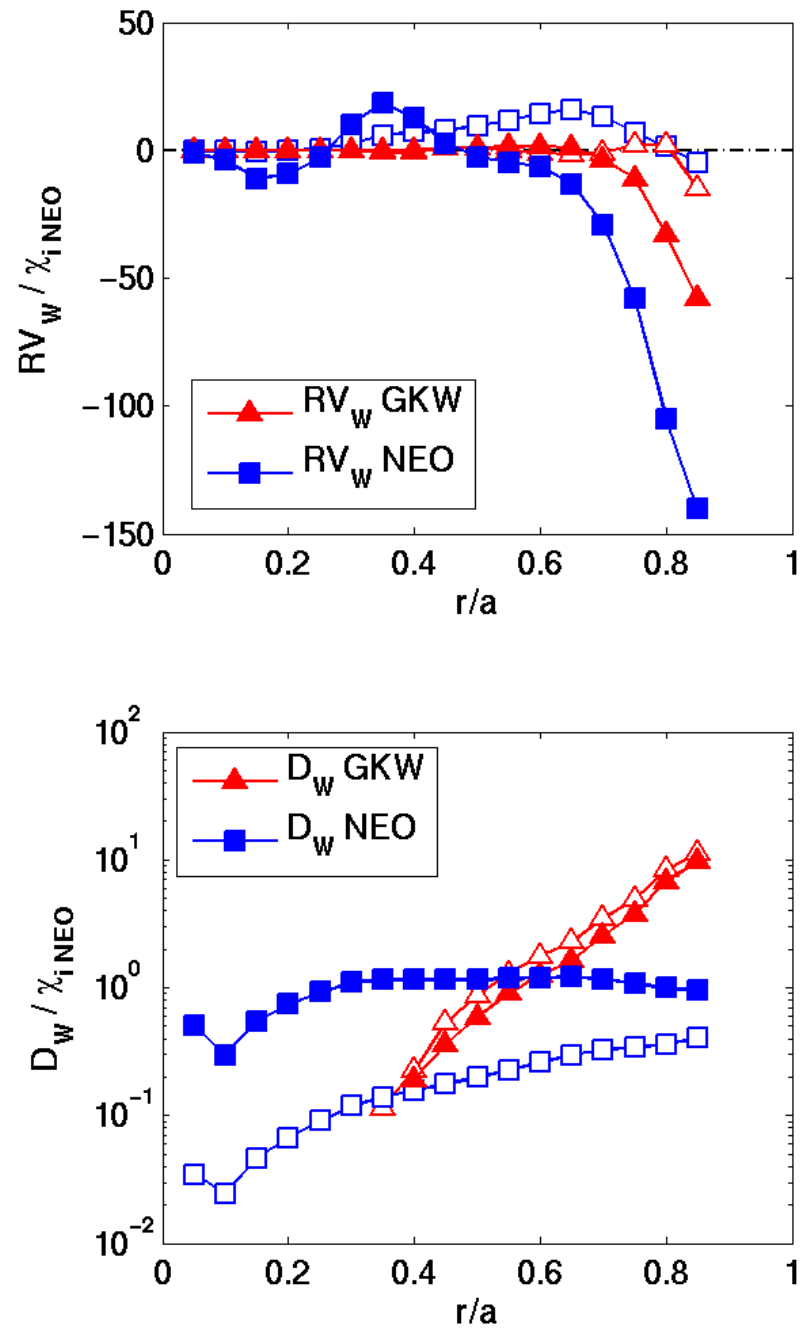

FIG. 9: W neoclassical and turbulent transport contributions computed by NEO and GKW respectively as they appear in Eq. (3) for the time slice at 10.42s. 

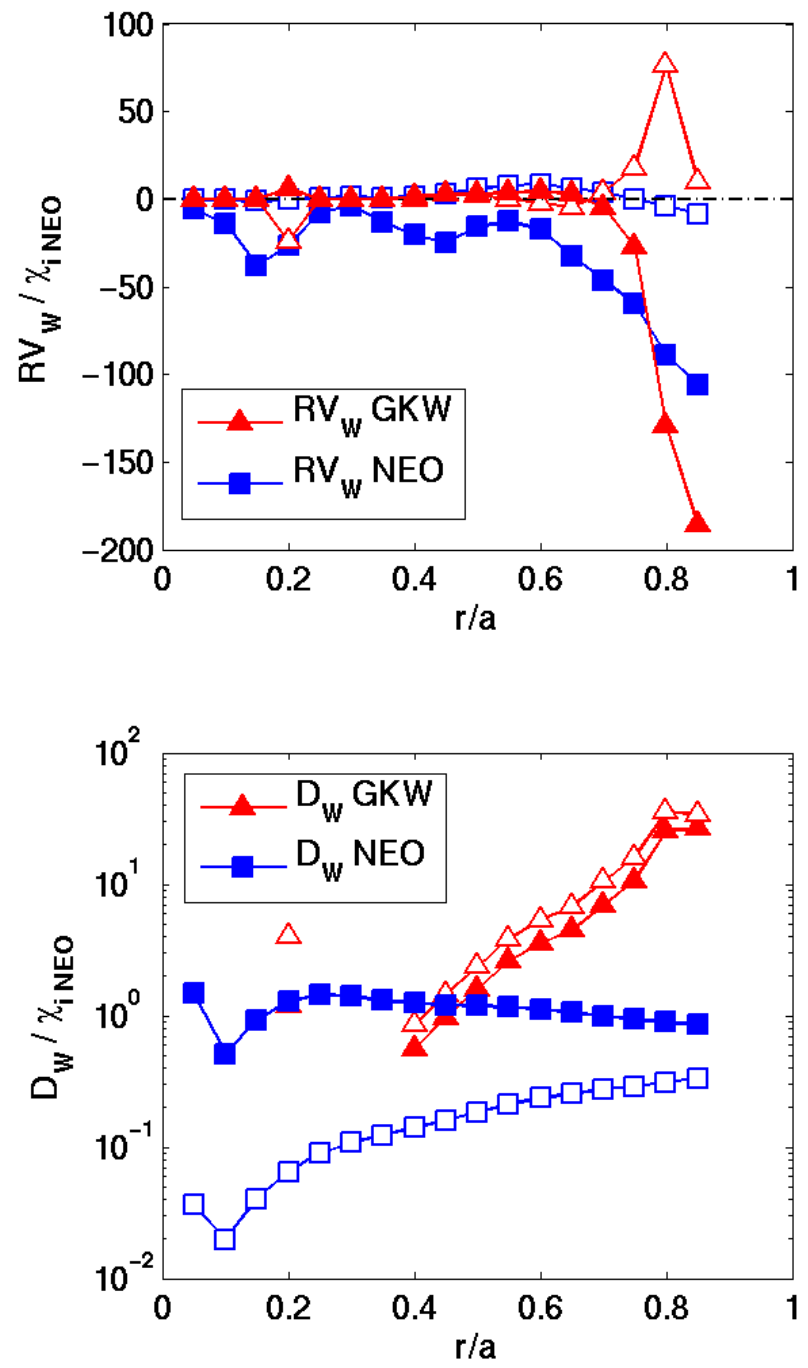

FIG. 10: W neoclassical and turbulent transport contributions computed by NEO and GKW respectively as they appear in Eq. (3) for the time slice at $12.37 \mathrm{~s}$. 

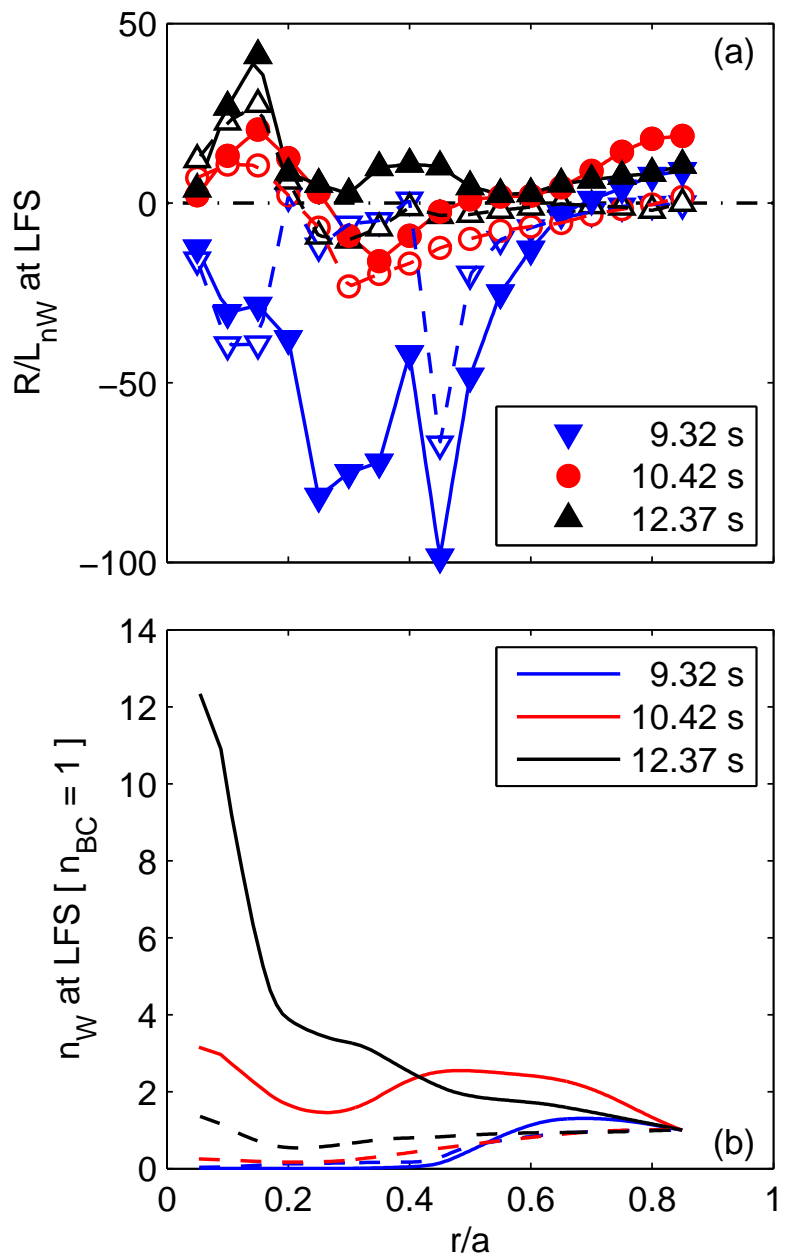

FIG. 11: Predicted LFS profiles of the normalized logarithmic gradient of the $\mathrm{W}$ density with (full symbols) and without (open) the inclusion of CF effects (a) and predicted LFS density profiles of $\mathrm{W}$ with (solid) and without (dashed) the inclusion of CF effects. The integration factor for the computation of the density profile has been set in order to have the density at the boundary equal to 1 for all of the profiles. 

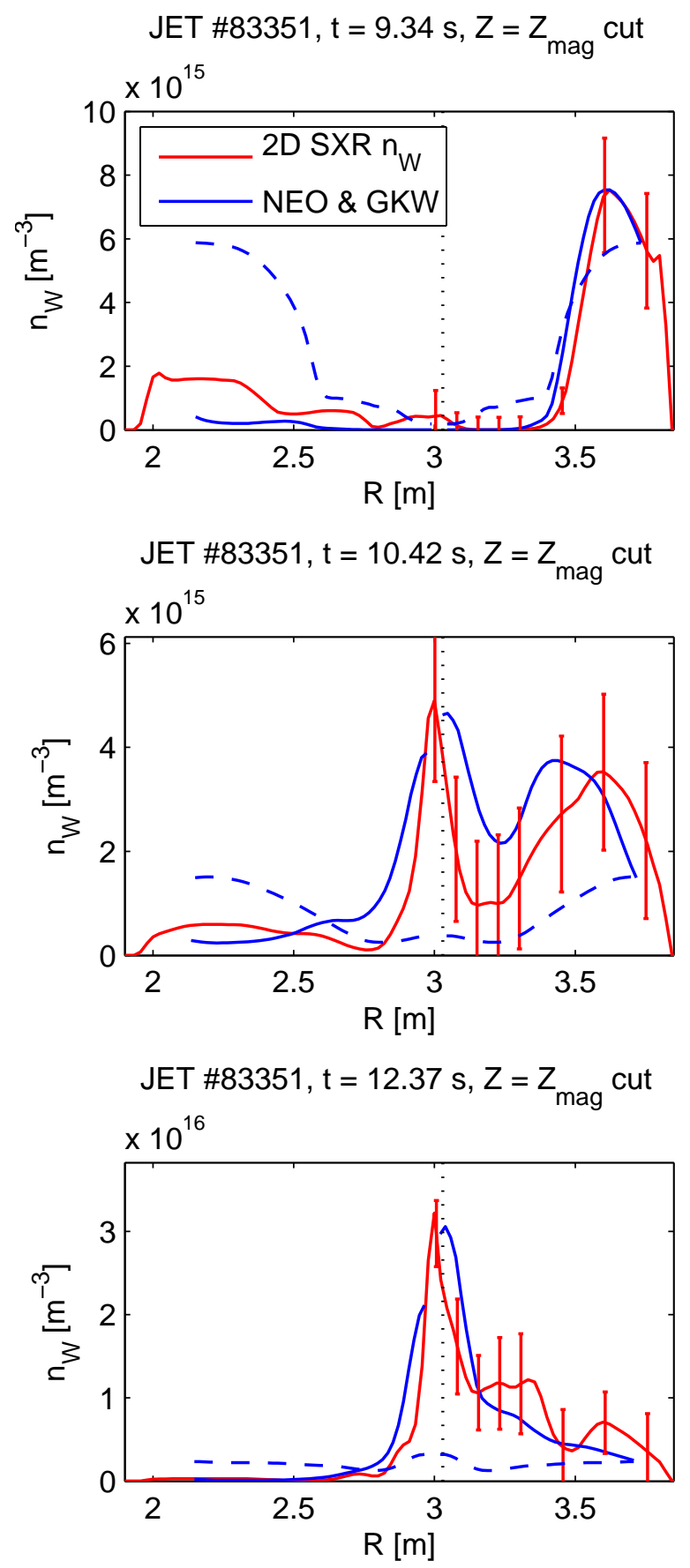

FIG. 12: Measured (solid with errorbars) and predicted (solid and dashed) W density as a function of the major radius in a horizontal cut at the height of the magnetic axis for shot \#83351 at 9.32s (a), 10.42s (b) and 12.37s (c). Predicted results with (solid) and without (dashed) the inclusion of CF effects in NEO and GKW are presented. 

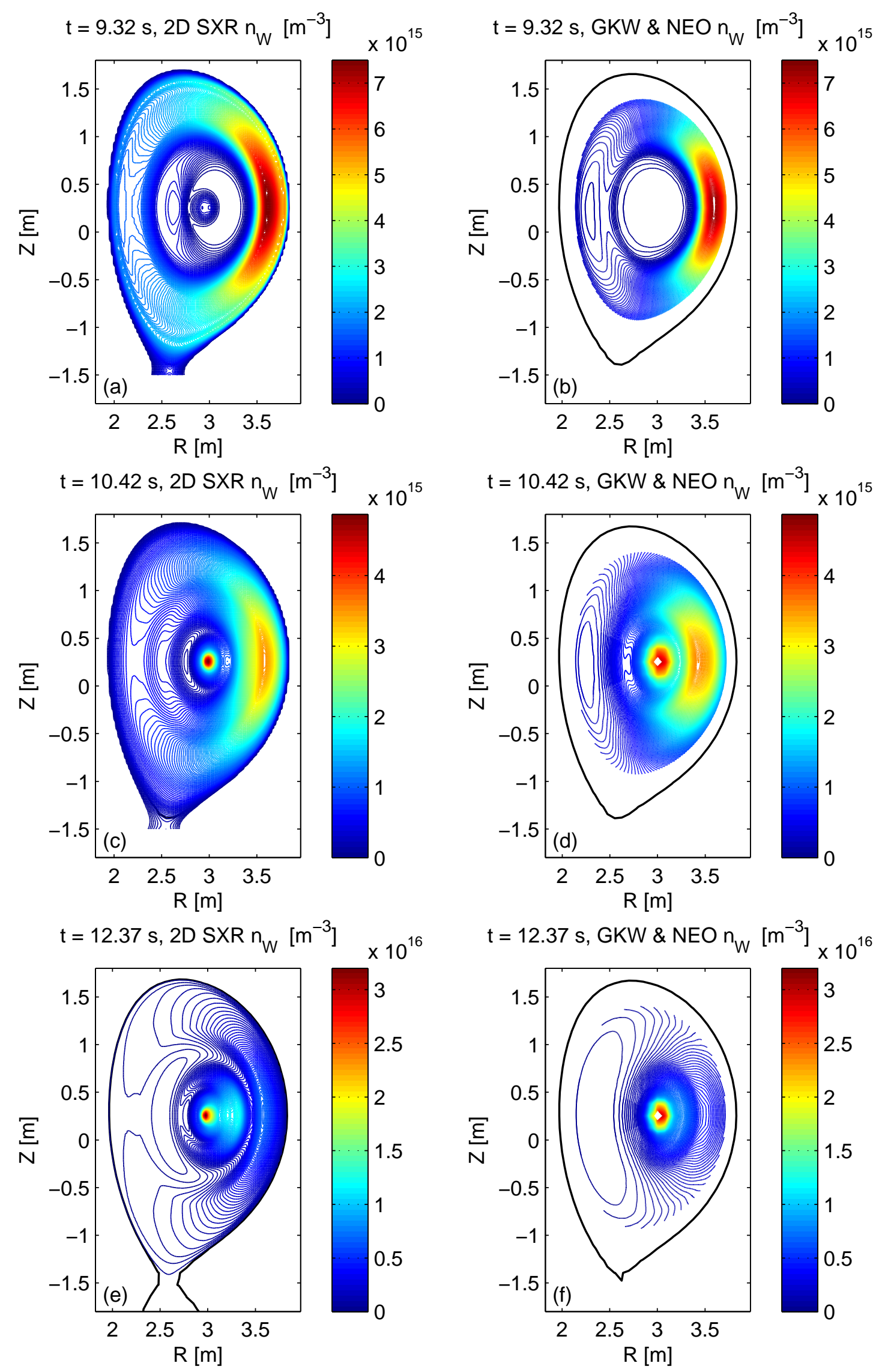

FIG. 13: Comparison of 2D W density distributions over the poloidal cross section obtained by means of an interpretive SXR diagnostics (left 26.8 lumn, a,c,d) and the predictions of the combined NEO and GKW simulations (right column, b,e,f), with the inclusion of CF effects. 